\title{
THE PATHOLOGICAL REPORT OF A CASE OF PHENYLPYRUVIC OLIGOPHRENIA
}

\author{
BY \\ J. A. N. CORSELLIS \\ From Runwell Hospital, Wickford, Essex
}

The urinary excretion of phenylpyruvic acid by mental defectives was first described by Følling in 1934. Since then it has been found to occur in about $0.5 \%$ of all mental defectives living in institutions. The condition has been widely studied from the biochemical and from the genetic points of view but less attention has been paid to the pathology. There appear so far to be 11 cases recorded in which any such report has been made and in the earlier ones no significant abnormality was found in the central nervous system. More recently, however (Alvord, Stevenson, Vogel, and Engle, 1950), it has been suggested that myelination is affected and it may therefore be of interest to report a case which does not entirely support this view.

\section{Clinical History}

The patient, R.C., was a female idiot, aged 25 at death. She was first admitted to Littlemore Hospital, Oxford, at the age of 7 with the report of having been mentally defective from a very early age. At the time of her admission in 1934 she was described as a well nourished child with straw-coloured hair and blue eyes. She had a broad palate and no ear lobules but apart from slight scarring at the angle of the mouth no skin abnormality was present, nor was any subsequently noted. She was epileptic, having both major and minor fits at frequent but irregular intervals. She was doubly incontinent. Her highest achievement was to feed herself and she could recognize her own name. On examination the lower limbs were slightly spastic and her gait was unsteady. All her voluntary movements were clumsy and uncoordinated.

In other respects her physical state appeared normal but in 1936 she was found to be excreting phenylpyruvic acid in the urine and this was consistently present in subsequent tests. Her blood Wassermann reaction on admission was negative.

From the time of her admission until her death 18 years later her mental state remained much the same-for the most part a cheerful idiot but at times a vicious one. She was inaccessible, untrainable, and resisted all examination. After the first few years the epilepsy was well controlled by phenobarbitone and no further fits occurred.

Her physical growth proceeded normally; her weight increased, menstruation started when she was 15 , and breast development was normal. In 1950 she developed lobar pneumonia which rapidly responded to penicillin and for two years she was in good health. In February, 1952, a similar attack occurred which again responded at once with complete recovery. Three months later, however, on May 4, she developed moderate pyrexia $\left(101^{\circ} \mathrm{F}\right.$.) with slight facial twitching but she could not be examined. At this time the blood picture was normal and the blood urea concentration was 28 mg./100 ml. Four days later (May 8) the temperature had increased to $103^{\circ}$. A lumbar puncture produced a normal cerebrospinal fluid under normal pressure. A few hours before death on the following day the temperature rose to $106^{\circ}$.

The patient was the second of four children; the eldest was a boy and then followed three girls. The youngest child at the time of examination was 2 years old and appeared normal. The other three children were all defective, the boy an imbecile, and the two girls (one the patient) were both epileptic idiots and excreted phenylpyruvic acid in the urine.

There was no history of mental defect, insanity, or epilepsy in either the parents or grandparents, or as far as is known, in any member of these preceding generations.

Post-mortem Examination.-This was carried out by Dr. Hagedorn 17 hours after the patient's death, and I am very grateful to him for his report.

The body was that of a well nourished girl.

A small amount of fluid was present in the pericardial sac. The heart was moderately dilated, with consequent thinning of the wall of the left ventricle. Microscopically the myocardium showed a mild degree of parenchymatous degeneration.

The thyroid appeared normal microscopically.

The lungs showed slight pulmonary emphysema.

A small amount of fluid was present in the peritoneal cavity. The spleen was slightly enlarged and soft ; microscopically the pulp appeared moderately congested. The liver showed slight parenchymatous degeneration 
microscopically. All other organs appeared normal to the naked eye.

It was noted that the skull-cap was unusually thick. The brain and spinal cord were removed and fixed in $10 \%$ formalin.

The weight of the fixed brain was 1,175g. Over the convexity, the general appearance of the meninges and blood vessels and the configuration of the gyri were normal. At the base, however, there was some localized swelling of the right hippocampal gyrus and uncus. A coronal cut made through the hippocampal gyri at this level did not show any anatomical abnormality.

In the right occipital lobe there were a few small (about $5 \mathrm{~mm}$. across), coalescing patches of greyish discoloration of the white matter lying caudal to the posterior horn and beneath the cortex of the convexity (Fig. 1).

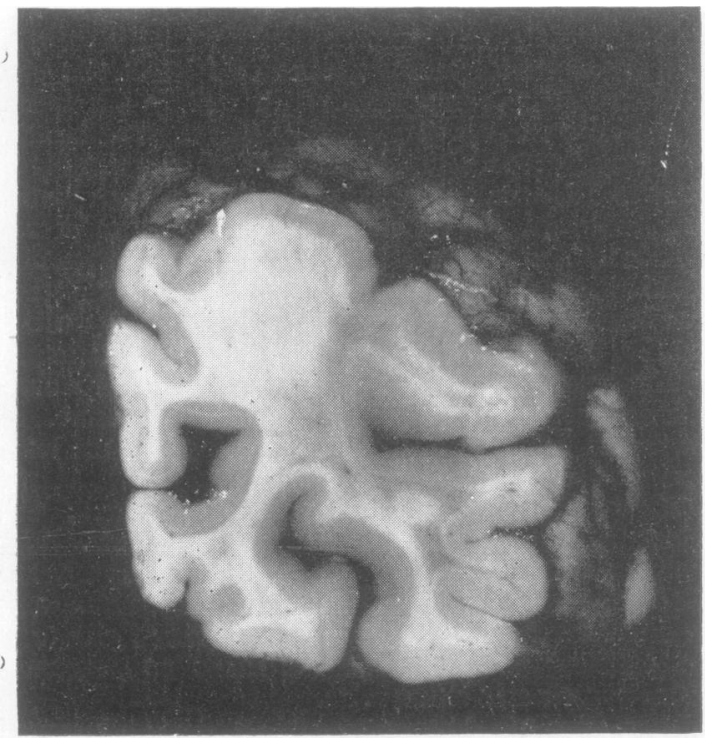

FIG. 1.-A few greyish patches in the white matter underlying the cortex of the convexity of the right occipital lobe.

The macroscopic appearance of the brain stem and spinal cord was normal but on both sides of the cerebellum there were patches of dark-brown staining of the meninges and of the underlying folia and white matter.

Microscopical Appearances.-Celloidin blocks were prepared from the left frontal areas and the left motor cortex ; from both occipital and both temporal lobes to include Ammon's horn. Bilateral blocks were taken from the basal ganglia, the optic chiasma, the midbrain, pons, medulla, and both sides of the cerebellum. Blocks from the spinal cord were taken at mid-cervical, mid-thoracic, and the twelfth thoracic levels.

Sections were stained with cresyl violet, haematoxylin and eosin, iron haematoxylin and van Gieson's counter- stain, phosphotungstic acid haematoxylin, by Heidenhain's method for myelin, and by Holzer's method for glial fibres. Frozen sections from representative areas were stained by Hortega's silver carbonate method for astrocytes, Bielschowsky's method for neurofibrils, Penfield's method for microglia, Herxheimer's method for fats, and by a modification of the Weigert-Pal method.

Frontal Areas.-There was very slight fibrous thickening of the leptomeninges. The molecular layer of the cortex showed a slight patchy increase in glial cells with some marginal gliosis. The structure and the lamination

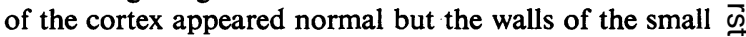
perforating blood vessels were unusually prominent $\bar{O}$ owing to the increased cellularity of the adventitia and slight adventitial fibrosis.

Throughout the cortex the nerve cells showed an almost complete loss of Nissl substance, resulting in a faintly stained homogeneous cytoplasm. The myelin of sheaths both in the cortex and deep in the centrum $\vec{O}$ ovale showed no abnormality.

Central Area.-In addition to the above findings most of the Betz cells were strikingly abnormal in that the nucleus was displaced to the periphery and showed an oval or irregular outline. The cell body tended to be rounded and often swollen with a peripheral ring of $\vec{\sigma}$ finely granular Nissl substance and a pale, more homo $\dot{\omega}$ geneous centre (Fig. 2). In sections stained with Scharlac $\vec{\omega}$ $\mathbf{R}$ some of the cells showed a small amount of yellow pigment lying at the extreme periphery. The appearance्s 을 in fact was similar to that seen in retrograde degenera tion, but there was no evidence of damage to the nerve fibres. A small minority of the Betz cells were shrunken and deeply stained while a few were ghostlike. None was normal.

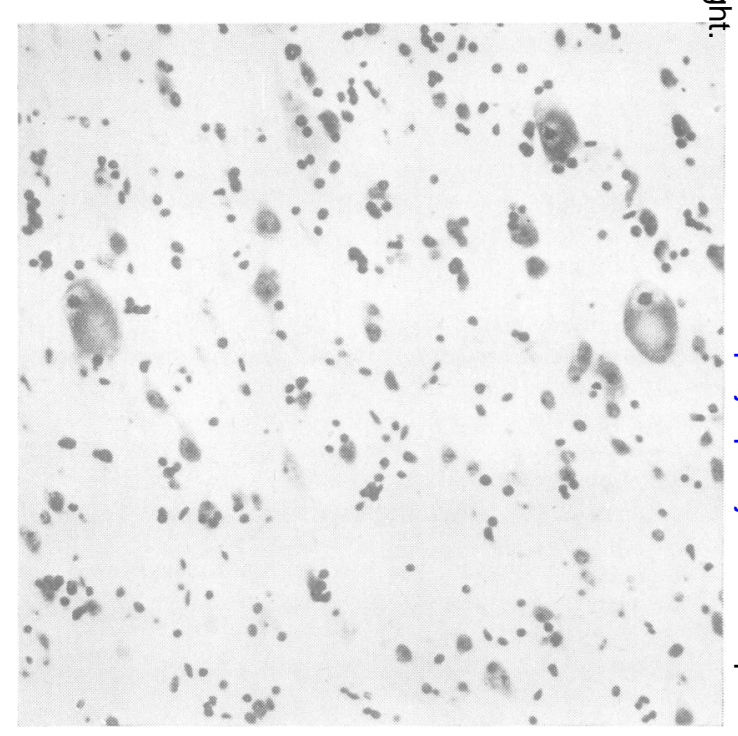

Fig. 2.-Two Betz cells with the typical appearance of retrograde degeneration. Cresyl violet $\times 230$. 
Occipital Lobes.-In general the appearances were similar to those in the frontal areas. In the right occipital lobe, however, there were several small patches of demyelination. The edge of these areas was not sharply defined and it tended to stop short of the arcuate fibres. There was considerable loss of myelin within the lesions while the remaining sheaths were beaded and fragmented. There was a tendency for the demyelination to be less severe around the blood vessels (Fig. 3). There

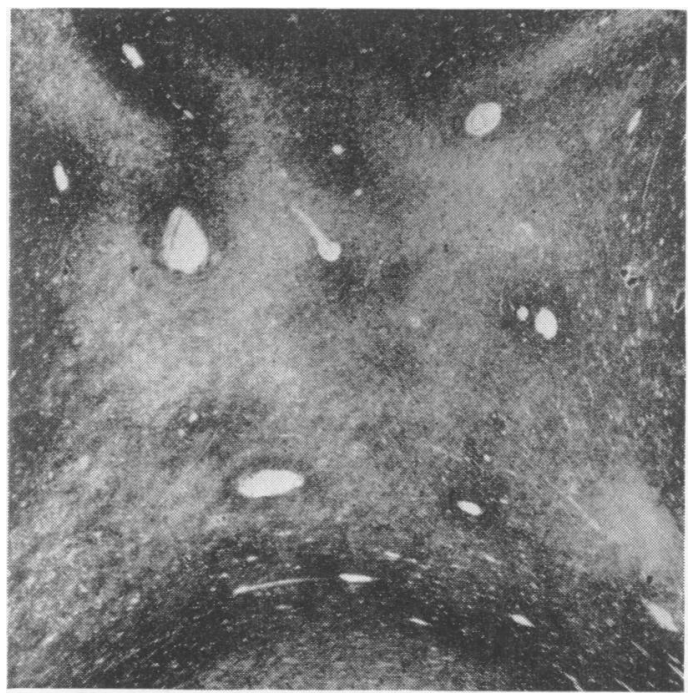

FIG. 3.-One of the small areas of demyelination in the white matter of the right occipital lobe. Kultschitsky Pal stain $\times 20$.

was only a very small number of foam cells containing lipoid stained by Scharlach $R$ but very considerable proliferation of astrocytes had occurred with an intense isomorphic gliosis. There was considerable loss of axis cylinders within the demyelinated areas and many of those remaining were thickened and swollen.

Temporal Lobes. - In the Sommer sector of both sides there appeared to be some slight paucity of pyramidal cells both diffusely and in a number of more localized patches, but there was no appreciable gliosis.

Striatum, Pallidum, and Thalamus. - In these areas and throughout the brain stem the walls of the smaller vessels were thickened by adventitial fibrosis and occasionally some hyaline change had taken place.

An occasional cluster of nerve cells in the thalamus showed " retrograde " changes but this was not restricted to any particular group. The nerve cells in the corpus striatum were normal. The myelination both of the pallidum and of the internal capsule was normal.

Mammillary Bodies. - The sub-pial surface showed a more intense fibrous gliosis than that seen elsewhere and many of the nerve cells lying just lateral to the mammillary body showed the appearance of retrograde degeneration. The main mammillary nuclei were normal and there was no vascular abnormality beyond that found elsewhere.
Mid-brain.-The nerve cells of the substantia nigra appeared to contain rather small amounts of pigment. The third and fourth nerve nuclei were almost lacking in Nissl substance but otherwise appeared normal.

Pons and Medulla.-Many of the nerve cells-often in groups-in the pontine nuclei showed the retrograde type of degeneration (Fig. 4 ), as did a considerable

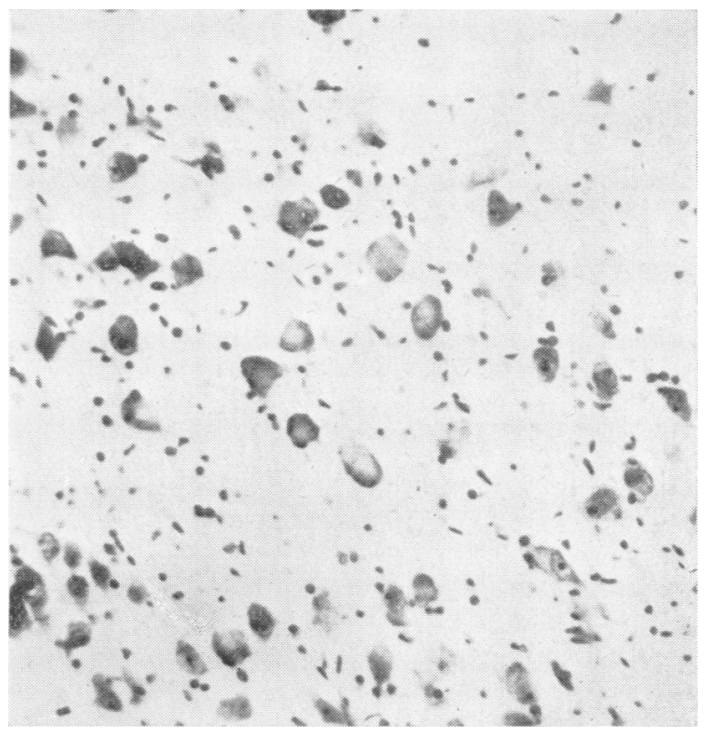

FIG. 4.-Nerve cells in the nuclei pontis showing the appearance of retrograde degeneration. Cresyl violet $\times 230$.

number of the smaller nerve cells scattered through the dorsal part. This was also noticeable in the reticular substance of the medulla, and, for example, in Roller's nucleus. Apart from the almost complete absence of Nissl substance the motor nuclei of the cranial nerves were mostly unaffected. The nucleus ambiguus (Fig. 5),

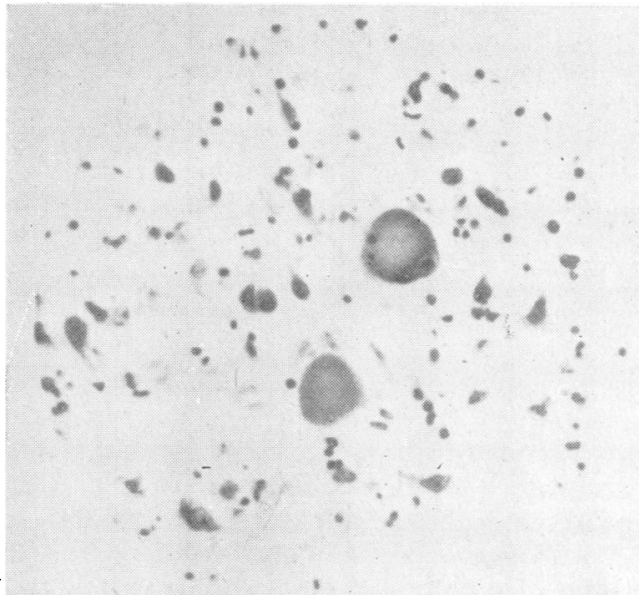

Fig. 5.-Nerve cells in the nucleus ambiguus. Cresyl violet $\times 230$. 
however, and to a lesser extent the dorsal nucleus of the vagus and the lateral cuneate nucleus all showed the type of cell change described.

Cerebellum.--On the meningeal surface of the cerebellum on both sides a few small areas of haemorrhage had occurred and the molecular layer of the underlying folia showed considerable vascular and microglial proliferation. There were a few small areas of necrosis in the subjacent granular layer. Elsewhere the cerebellum, including the Purkinje cells, the dentate nuclei, and the white matter, was normal.

Spinal Cord.-No signs of myelin defect were detected. In the mid-cervical and mid-thoracic segments a small number of anterior horn cells showed retrograde degeneration but many others were shrunken, with pyknotic nuclei and darkly stained cytoplasm. There was some fibrosis and hyalinization of the small vessels both within the cord and in the surrounding meninges.

\section{Discussion}

In previously reported cases nothing very similar to the present findings has been described. In the first pathological report (Penrose, 1939) the main feature was the presence of multiple neurofibromata of the peripheral nerves with some associated loss of myelin but with the central nervous system virtually intact. In the second case (Coquet, Myle, Nyssen, and van Bogaert, 1944) a small necrotic area was found in the white matter of one frontal lobe but there were no further lesions of the myelin and the brain in all other respects was normal. Josephy (1948) investigated two cases and " after a rather careful microscopical examination of all organs and especially of the brain" found "no significant abnormality whatsoever".

In 1950 Alvord, Stevenson, Vogel, and Engle examined the brains of two siblings who died at the age of 16 months and $5 \frac{1}{2}$ years respectively. The first died from staphylococcal septicaemia after almost a year of severe illnesses. The second died in hyperpyrexia two weeks after the onset of measles complicated by pneumonia. Both these cases, but especially the first, showed evidence of a retardation of myelin formation. In three further adult cases no similar myelin defect was found, but in one there was a marked loss of myelinated fibres in the centre of the optic chiasma. On the basis of their evidence the authors put forward the suggestion that both retarded myelination and also demyelination are features of this condition. They further postulated that these findings might, on the one hand, be at least partly due to the metabolic anomaly present in phenylpyruvic oligophrenia, and on the other hand, be at least partly responsible for the mental deficiency which is also invariably present.
This report was followed more recently by tha $\bar{Z}$ of Benda (1952) in which he described the findings if two further cases; both patients were aged $2 \overrightarrow{\mathrm{p}}$ and both had died from tuberculosis. In these cases the appearances were not of incomplete myelination but consisted rather of widespread ando " marked damage to the myelin sheaths and white matter of the brain ". In both of them, however, Benda pointed out that the relationship between the demyelination and the metabolic abnormality wa uncertain. He considered, but partly in view of? Alvord's report was inclined to reject, the suggestion that prolonged tuberculosis might be a significan factor. (It is interesting to note that one of the patients described by Josephy died of tuberculosis but showed no abnormality.) He further considereç that the findings described by Alvord and others madethe case for accepting the metabolic abnormality as the cause of the myelin defect very strong. It seems possible, however, that these findings might have also been related to other factors ; for instance, as series of severe and prolonged infections in the infant and death during measles in the child $\vec{\rho}$ Moreover, even if myelination is retarded in earuy life there appears to be no evidence that demyelipa $\overrightarrow{.}$ tion would therefore be a more likely finding in adult.

With regard to the white matter of the present case, there was certainly an irregular patch demyelination situated in the most posterior limitso one occipital lobe. Apart from this, however, other damage to the myelin was found and a probs able cause of the lesion was the epilepsy in the earlier years of life. In any case it does not seemp reasonable to regard this one restricted patch of heavily gliosed demyelination as the direct mani festation of a metabolic disorder present throughouf life. In the same way the normal appearance of the white matter in all other respects gives no support to the suggestion that retarded formation is क constant feature of this disease-at any rate of at degree sufficient to show at the age of 25 .

In view of the findings in this case and from the evidence of other case reports (with the possible exception of those of Benda), it therefore appear? that demyelination has so far been neither a constan $\mathrm{B}$ nor an outstanding feature. Furthermore, while retarded myelination has also been described it is difficult to accept the argument that such retardation could " be at least in part responsible for the mentap deficiency" and yet for it to be undetectable in the young adult. Only the examination of further cases can settle these points.

Turning now to a closer discussion of the present case, and especially the abnormal appearance of the 
nerve cells, a number of possibilities must be considered. At the same time there is no point in considering them other than very briefly since too little is known about either the cause of the patient's pyrexia or of her death. The almost complete absence of Nissl substance may be accounted for by the terminal state. The appearance of retrograde degeneration, however, with the present distribution and in the absence of visible damage to the related nerve fibres, is a completely separate finding and cannot be dismissed in this way. Certainly the most likely cause of such a finding is the presence of complicating disease. In the circumstances the immediate suspicion is that the patient was pellagrous since this is a condition which, in spite of all attempts to prevent it, tends to occur among mental patients and which produces the neuronal changes, both in appearance and in distribution, and the vascular changes peculiarly like those found in the present case (Leigh, 1952). The strongest argument against the diagnosis is that there was no suggestion clinically of a pellagrous state. There was no loss of weight, there were never any skin lesions, and although the patient was incontinent, there was no manifest diarrhoea. On the other hand the absence of evidence in such a patient may not be conclusive since little more than veterinary contact with her was ever possible. It seems therefore that while a pellagrous condition cannot be excluded the clinical evidence in favour of its existence is not great. In much the same way, apart from the pyrexia, no evidence of an acute infection was found. In fact all that is known for certain is that the patient became pyrexial and died. In these circumstances it seems futile to try any further to identify the cause of the neuronal changes although they may prove to be of sufficient interest to justify their description.

There is of course the possibility that the nerve cell changes are more directly related to the biochemical abnormality present but this seems improbable. In none of the previous cases have such changes been reported, and in fact Benda commented on "the surprising observation that the nerve cells in phenylpyruvic oligophrenia appear quite well preserved in many places and especially the Nissl substance is quite conspicuous in the anterior horn cells."

From the speculative point of view there are two points which may be mentioned. First, there is some superficial connexion between the nutritional deficiencies, such as pellagra, and the condition of phenylpyruvic oligophrenia. In both cases there is a metabolic disturbance but, while in the former this is due to a vitamin lack, in the latter it is probably due to the absence of a particular enzyme system. The second point is that the appearance of retrograde degeneration may well be related to an abnormality of protein metabolism, and, since phenylpyruvic oligophrenia is essentially due to a fault in the metabolism of phenylalanine, it is conceivable that a link may exist.

\section{Summary}

The pathological findings in a case of phenylpyruvic oligophrenia are described. These consisted of one rather limited area of demyelination but there was no evidence of abnormality of myelin formation.

Marked " retrograde" nerve cell changes were seen in the Betz cells and in certain other groups of nerve cells in the brain stem and spinal cord.

The question of myelin abnormality in this condition is discussed and possible explanations of the nerve cell changes are briefly mentioned.

I am very grateful to Dr. R. W. Armstrong, the Physician Superintendent, and to Dr. E. O. Hagedorn of Littlemore Hospital, Oxford, for permission to make use of the clinical records and for the post-mortem material of this case. I am extremely grateful to Dr. J. G. Greenfield and to Professor Alfred Meyer for their help and advice in the preparation of this report.

\section{REFERENCES}

Alvord, E. C., Stevenson, L. D., Vogel, F. S., and Engle, R. L. (1950). J. Neuropath., 9, 298.

Benda, C. E. (1952). Developmental Disorders of Mentation and Cerebral Palsies. Grune and Stratton, New York.

Coquet, M., Myle, G., Nyssen, R., and Bogaert, L. van (1944). Mschr. Psychiat. Neurol., 109,'133.

Folling, A. (1934). Nord. med. T., 8, 1054.

Josephy, H. (1948). Illinois Med. J., 94, 107.

Leigh, D. (1952). J. ment. Sci., 98, 130.

Penrose, L. S. (1939). Lancet, 1, 572. 